Pteridines

Vol. 22, 2011, pp. $18-23$

\title{
Effects of Inhalation Anesthetics on the Profile of Urinary Neopterin as an Immune Marker in Patients who Underwent Laparoscopic Cholecystectomy
}

\author{
Zeynep Capan ${ }^{1}$, Terken Baydar², Gozde Girgin², Anil Dolgun², Beyazit Dikmen', Gonul Sahin² \\ ${ }^{1}$ Anesthesiology and Reanimation Clinic, Ankara Hospital, Ankara, Turkey, ${ }^{2}$ Department of Toxicology, Faculty \\ of Pharmacy, and ${ }^{3}$ Department of Biostatistics, Hacettepe University, Ankara, Turkey
}

Accepted: 2011/04/13

\begin{abstract}
It has been claimed that inhalational anesthetics affect many phases of immune response. Although, there are a lot of studies on the effects of widely used anaesthetic agents and methods on the immune system, there are only a few studies to show the effects by evaluation of neopterin concentrations as an important marker of cellular immunity. This study was done to evaluate possible effects of inhalation anesthetics, such as desflurane, isoflurane, and sevoflurane on the immune system by measuring urinary neopterin concentrations in patients who underwent laparoscopic cholecystectomy. The study is randomized, prospective and double blind. Urinary neopterin was measured in patients submitted to general anesthesia with either desflurane $(n=20)$ or isoflurane $(\mathrm{n}=11)$, or sevoflurane $(\mathrm{n}=13)$ in laparoscopic cholecystectomy. Urine samples were collected preoperatively, at $2^{\text {nd }}$ and $24^{\text {th }}$ hours post-operatively and the neopterin per creatinine concentrations were measured by using high-performance liquid chromatography.

The preoperative neopterin levels were similar across the study groups. Variance analysis of repeated measures showed an effect of time $(\mathrm{p}<0.05)$ : Neopterin concentrations increased over time, but the effect of different anesthetics on neopterin was similar. The mean increases in neopterin concentrations were similar among all the applied anesthetics. In conclusion, this report shows changes of immune response by investigation of neopterin in urine samples during 24 hours in patients exposed to three different inhalational anesthetic agents sevoflurane, desflurane and isoflurane in laparoscopic cholecystectomy.
\end{abstract}

Key words: neopterin, sevoflurane, desflurane, isoflurane, inhalational anesthesia, laparoscopic cholecystectomy

\section{Introduction}

High infection rates in the postoperative period, negative effects of anesthetics on the patients suffering from immune deficiency syndromes such as AIDS and the high incidence of spreading of malign cells in cancer patients have speeded up the studies on the effect of surgery and anesthesia on the human immune system (1). Effects of anesthetics on immune system were first studied with ether (2), and the following numerous studies related to various anesthetics revealed effects on this system. It has been found that especially volatile and intravenous anesthetics depress immune cell functions. The studies until 1990's were directed towards the negative effects of immune suppression due to anesthetic agents or anesthetic mechanisms. In spite of the fact that many immunological effects of anesthetics have been better understood in the recent years, the mechanisms and clinical importance of these effects are still unclear (3). It also remains uncertain how anesthetics may influence morbidity and mortality. It has been suggested that inhalational anesthetics depress chemotactic, phagocytic and microbicidal activities of neutrophils and monocytes (4).

There is a limited number of studies about the effects of anaesthetics on immune system in patients without having surgical trauma. In a study comparing general anesthesia and epidural anesthesia it was reported that in the absence of surgery, general epidural anesthesia has only little effect on the immune system (5). According to the findings, there is a temporary immune depression after anesthetic management

Correspondence to: Prof.Gonul Sahin, Ph.D., Toxicology Department, Faculty of Pharmacy, Hacettepe University, TR90-06100, Ankara, Turkey. Telephone: +90 312305 2178, Fax: +90 312311 4777,

E-mail: gsahin@hacettepe.edu.tr

Pteridines/Vol. 22/No. 1 
including decrease in the activity of natural killer (NK) cells, and in the count of $\mathrm{T}$ and $\mathrm{B}$ lymphocytes and suppression of proliferative response to mitogens and decrease in neutrophil oxidative responses (5-9). Anesthetic agents especially together with surgical stimuli, have either direct or hormone-dependent effects via hypothalamo-hypophyseal-adrenal (HHA) axes on immunologic mechanisms. In the perioperative period, all of the neuroendocrine system, anti- and pro-inflammatory cytokines cause an increase in their suppressive effects. In in vivo studies, the main difficulty is to distinguish the specific effects of the anesthetic agent from those resulted from the other multiple intraoperative factors. These factors include type and duration of surgery, blood transfusion, basal immunological state and body temperature. Although it seems likely that surgery is associated with significant suppression of postoperative immune function; the degree depends primarily on the type and duration of the procedure. Therefore, the results of the studies cannot be easily applied in clinical practice $(10,11)$.

T-lymphocyte changes in operated patients can be seen as delayed type hypersensitivity (DTH) responses. DTH is a multifactorial response which contains both lymphocyte and lymphocyte subpopulation interactions; they can affect final responses. T cell-related immune function is used to assess DTH response. Since laparoscopic surgery reduces the magnitude of surgical trauma, it is thought to be associated with less immune impairment including decrease in T-cell function (12-14). disorders (15-18). Many components of the immune system in the surgical patients under anesthesia were evaluated by measuring T-cell subpopulations like $\mathrm{CD}^{+}$and $\mathrm{CD} 8^{+} \mathrm{T}$-cells, serum levels of $\beta_{2}$-microglobuline, of cytokines like interleukin-2 (IL-2), IFN- $\gamma$, and tumor necrosis factor-alpha (TNF- $\alpha)(3,5,11)$. However, the isolation and measurement of these markers in biological fluids is difficult, variable, and expensive; and their application in clinical practice it is not always feasible. Although blood and/or even urinary neopterin serves as a sensitive and early biomarker of cellular immune system activation, the relationship between neopterin release and exposure to anesthetics has not been assessed in greater detail with only a few exceptions $(19,20)$. Therefore, the main goal of the present study was undertaken to investigate possible effects of inhalation anesthetics, such as desflurane, isoflurane, and sevoflurane on immune system by measuring urinary neopterin in patients who underwent laparoscopic cholecystectomy.

\section{Materials and Methods}

This report results from a randomized, prospective and double blind study, performed in Ankara Hospital, Anesthesiology and Reanimation Clinic between January and December 2009. It was in concordance with the Helsinki Declaration 1981 and approved by the ethic committee. The demographic data of the patients are listed in Table 1.

Table 1. Demographic data of the patients. *Mean value \pm standard deviation (minimum - maximum)

\section{Groups \\ Number}

Sevoflurane (S)

Isoflurane (I)

Desflurane (D)
13

20
Gender

(Female/Male)

$17 / 3$
Age as year

$$
\begin{aligned}
& 44.2 \pm 11.3(23-56)^{*} \\
& 42.4 \pm 14.3(26-62) \\
& 38.8 \pm 8.21(27-45)
\end{aligned}
$$

Measurement of neopterin levels in non-invasive samples provides information about activation of cellular immune system under the control of type $1 \mathrm{~T}$ helper (Th1) cells. Neopterin is produced primarily by human monocytes/macrophages upon stimulation by interferon- $\gamma$ (IFN- $\gamma$ ), and also it is an indicator of a proinflammatory immune status. Increased neopterin is found in infections by viruses such as human immunodeficiency virus (HIV), intracellular living bacteria and parasites infections and in many diseases, i.e. autoimmune diseases, malignant tumors, allograft rejection episodes, neurological and cardiovascular
According to the American Society of Anesthesiologists grading, 44 ASA I-II graded patients scheduled to have laparoscopic cholecystectomy were randomly allocated to three groups (13 patients to sevoflurane, 20 patients to desflurane and 11 patients to isoflurane groups), each planned to have different inhalational anesthetic agents for the maintenance of the anesthesia. Patients with systemic chronic inflammatory and coronary artery diseases, or malignancies, and smokers, alcoholics and drug abusers were excluded from the study. Patients did not receive any premedication. The peripheral oxygen saturation $\left(\mathrm{SpO}_{2}\right)$, electrocar- 
diogram (ECG) and non-invasive blood pressures were monitored (PM 8060 Vitara, Drager, Germany).

As preoperative samples, urine samples were collected 15 minutes before anesthetic induction (Baseline). Afterwards, all patients received $5 \mathrm{mg} / \mathrm{kg}$ thiopenthal, $0.1 \mathrm{mg} / \mathrm{kg}$ vecuronium and $1 \mu \mathrm{g} / \mathrm{kg}$ fentanyl for induction and nitrous oxide $50 \%$, oxygen 50 $\%$ with either sevoflurane $2 \%$ (Group S) or desflurane $6 \%$ (Group D) or isoflurane $1.5 \%$ (Group I) for the maintenance. Additional medication was not given to patients except additional neuromuscular blockers. After the end of the surgery, the residual effects of neuromuscular blocker were antagonized with neostigmin $2.5 \mathrm{mg}$ and atropine $0.5 \mathrm{mg}$, and the patients were extubated. As postoperative samples, urine samples were taken at the $2^{\text {nd }}$ and at $24^{\text {th }}$ hours after operation. Urine specimens were kept from direct light, and stored at $-20^{\circ} \mathrm{C}$ until the assays were performed.

Neopterin concentrations were determined by highperformance liquid chromatography (HPLC) using a fluorescence detector (HP, Agilent1100) at a wavelength of $353 \mathrm{~nm}$ for excitation and $438 \mathrm{~nm}$ for emission, as described before (21-24). Creatinine concentrations were detected simultaneously by HPLC using an ultraviolet detector (HP, Agilent1100) at the wavelength of $235 \mathrm{~nm}$. The neopterin levels were given as micromoles of neopterin per mol of creatinine.

Baseline neopterin levels of the three anesthetic groups are compared using one way analysis of variance. Repeated measures analysis of variance is used to analyze the effect of anesthetic groups on the neopterin levels which are measured at 3 different time points (baseline, 2 hours and 24 hours after the operation). A total of 44 patients achieved $99.6 \%$ power to detect whether the time effect is statistically significant. All statistical analyses were performed using the SPSS program (version 15.0 for Windows), and statis- tical significance was set at $\mathrm{p}<0.05$.

\section{Results}

Mean neopterin levels of the three anesthetic groups at baseline, post1 (2 hours after the operation) and post2 (24 hours after the operation) are presented in Figure 1. The mean baseline neopterin levels were similar across the three anesthetic groups, D, I, and S

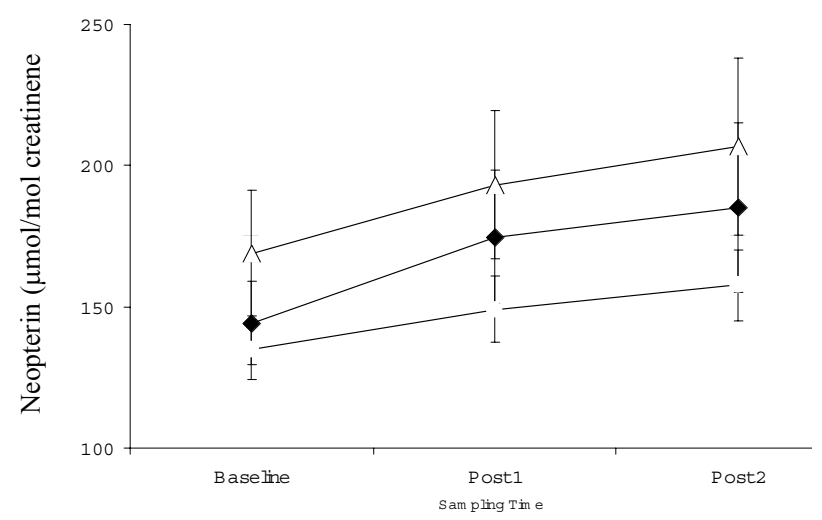

Figure 1. Mean neopterin levels of three anaesthetic groups. $\Delta$, Desflurane group; $\diamond$, Sevoflurane group;o, Isoflurane group. Baseline; post $1=2$ hours after the operation; post $2=24$ hours after the operation. The results are expressed as the mean \pm standard error (SEM).

$(\mathrm{p}=0.302)$.

Mean neopterin levels increased over time $(\mathrm{p}<0.05$, repeated measures analysis of variance). However, the effect of time was similar in all 3 groups of anesthetics ( $\mathrm{p}=0.933$; Figure 1$)$. The mean changes as a percentage in the urinary neopterin among three anesthetic groups are given in Table 2. Mean increases in

Table 2.: Percentages of mean changes in the levels of neopterin in 3 groups of patients receiving different anaesthetics ( $\mathrm{S}=$ Sevoflurane, $\mathrm{I}=$ Isoflurane, $\mathrm{D}=$ Desflurane).

\section{Percentage of changes in neopterin levels}

$\begin{array}{lccc} & \text { Baseline - Post } 1 & \text { Post } 1 \text { - Post } 2 & \text { Baseline- Post } 2 \\ \text { Group S, } \boldsymbol{n}=\mathbf{1 3} & +19.5 & +14.0 & +27.9 \\ \text { Group I, } \boldsymbol{n}=\mathbf{1 1} & +13.0 & +14.9 & +27.3 \\ \text { Group D, } \boldsymbol{n}=\mathbf{2 0} & +16.6 & +14.1 & +29.5 \\ \text { All patients, } \boldsymbol{n}=\mathbf{4 4} & +16.6 & +14.3 & +28.5 \\ \text { P values } & \mathbf{0 . 8 0 1} & \mathbf{0 . 9 9 9} & \mathbf{0 . 9 9 5}\end{array}$


neopterin levels were similar among the anesthetic groups ( $p<0.05$; one way analysis of variance).

\section{Discussion}

Today, isoflurane, desflurane and sevoflurane among many inhalational anesthetics are widely used in many surgical procedures in order to provide optimum surgical conditions for both to the patient and the surgeon. Beside some advantages of these agents, they may have untoward effects on many functions in the body including the immune system. In addition to this, the effect of surgical stress on the immune system should not be underestimated. There is still not an optimal approach in determining the immune status of patients under surgical-anesthetic stress (3, 11-14). Fortunately, during last two decades, laparoscopic surgery has become more popular than open surgical practice with the advantages of less postoperative pain, earlier recovery and better cosmetic outcomes, earlier discharge from the hospital and in addition to these it causes less tissue trauma then open operation. Thus, it is likely to be associated with better preservation of systemic immune function (12-14).

The effects of anesthetics and anesthetic methods on the human immune system are being investigated since the time of ether anesthesia and peaked with the studies performed on anesthesia workers (25). The immunosuppressant effects of anesthesia together with surgical trauma can play a relevant role in postoperative infection occurrence. In addition to surgery, factors such as plasma transfusion, hypothermia, hyperglycemia during perioperative period, and postoperative pain may decrease both humoral and cellular immunity. It is postulated that every anesthetic agent more or less suppress immunologic mechanisms (111). Immune response is a protective phenomenon against any foreign antigen, but due to the nature of the antigen, antibodies produced and patient's susceptibility, it may be deleterious $(15,16)$. Although there are a lot of clinical studies on the effects of widely used anesthetic agents and methods on immune system either in vivo or in vitro $(7-14,26,27)$, there is limited number of evidence on neopterin which is an important marker of cellular immunity $(20,25)$. Determination of neopterin concentrations in biological fluids seems to be more useful, non-invasive, cheaper and reliable (1517, 22-24). By considering the little effect of laparoscopic surgery on the immune system, the immunologic effects of three inhalation agents were evaluated by monitoring the urinary neopterin levels in this work in patients who underwent laparoscopic cholecystectomy. In this study, neopterin levels showed slight elevation in all study groups at 2 hours after the operation in comparison to baseline. Similarly, increasing tendency continued at 24 hours after the operation. However, the differences were not statistically significant. According to these results, it may be thought that the inhalational anesthetics in laparoscopic surgery patients caused alterations in neopterin levels as indicator of the cellular immune system. When effects of laparoscopic surgery on immune system are considered to be unimportant according to literature, this alteration may originate from exposure of the inhalational anesthetics. However, the results obtained from studies in which different type of surgical treatments were applied showed that there is no effect of the anesthesia agents on neopterin status in contrast to our suggestion $(19,28,29)$.

Our results also showed that there is not any superiority among isoflurane, desflurane, or sevoflurane. Many studies suggest that inhalational anesthetics interfere with many phases of the immune system: bone marrow depression, inhibition of phagocytosis and macrophage mobility. In a study performed by Propicio et al. in two groups in the absence of surgery, exposed healthy volunteers either to mask thiopenthalisoflurane-nitrous oxide anesthesia or lumbar epidural anesthesia and the immune function of sampled ex vivo cells from these volunteers were unaffected (5). Clearly, the interaction among the stressors of surgery the perianaesthetic period, and anesthetics agents account for the postoperative immune modulation (111). Moudgill et al. showed that isoflurane does not effect while halothane and enflurane significantly suppress neutrophil chemotaxis (7). On the other hand, sevoflurane is thought to have a little effect on neutrophil apoptosis, cytokine concentration and neutrophil count, but desflurane seems to cause greater systemic response effects $(26,27)$. Marfella et al. reported a marked decrease in neopterin level at 4 hours after anaesthetic induction, however they found an increase in neopterin levels at 24 hours in patients with uterine malignancies operated either halothane or isoflurane (20). In our study sevoflurane, desflurane, and isoflurane resulted in an increase urinary neopterin levels in the postoperative 2 nd hour $19.5 \%, 16.6 \%$, and $13.0 \%$, respectively. Moreover, it has been shown that the increase in neopterin levels continued from the $2^{\text {nd }}$ postoperative hour to the $24^{\text {th }}$ postoperative hour in both, the sevoflurane and the desflurane groups, with similar trends $(\sim+14 \%)$. Increase in neopterin levels of the isoflurane group was kept in a steady state. According to these results, it seems that sevoflurane and desflurane have reversible effects on the immune system. At the moment, it is difficult to say the same thing for isoflurane. It is not surprising to meet some conflict between our findings and results of the abovementioned studies because of the different study 
designs.

In conclusion, this paper reports alteration of immune system by evaluation of urinary neopterin in patients exposed to three different inhalational anesthetic agents during laparoscopic surgery. It seems that, depending to the type of the surgery, sevoflurane, desflurane and isoflurane have minimal effects on the urinary neopterin status. Finally, further studies are needed to solve unclear points.

\section{Declaration of conflicting interests}

The authors report no conflicts of interest. The authors alone are responsible for the content and writing of the paper.

\section{References}

1 Shorten GD. Potential adverse effects of volatile anaesthetic agents-Immune dysfunction and chronic occupational exposure. ESA refresher course lectures. Nice, In European Society of Anaesthesiologists (eds) 2002; p. 207-10.

2 Graham EA. The influence of ether and ether anaesthesia on bacteriolysis, agglutination and phagocytosis. J Infect Dis 1911; 8 (2): 147-75

3 Salo M. Effects of anaesthesia and surgery on the immune response. Acta Anaesth Scand 1992; 36: 201-20.

4 Adams DH, Nash GB. Disturbance of leukocyte circulation and adhesion to the endotelium as factors in circulatory pathology. Brit J Anaesth 1996; 77:17-31

5 Procopio MA, Rassias AJ, De Leo JA. The in vivo effects of general and epidural anesthesia on human immune function. Anesth Analg 2001; 93: 460-5.

6 Stevenson GV, Hall SC, Rudnick S. The effect of anesthetic agents on the human immune response. Anesthesiology 1990; 72: 542-52.

7 Moudgil GC. Update on anesthesia and the immune response. Can J Anaesth 1986; 33: 44-60.

8 Walton B. Effects of anesthesia and surgery on immune status. Brit J Anaesth 1979; 51: 37-43

9 Kumar A, Sethi AK. Anaesthesia-Immune system interactions: Implications for anaesthesiologists and current perspectives. Indian J Anaesth 2002: 46 (1): 8-20.

10 Celebioglu B, Ozer E. Anestezi ve immun sistem. Anestezi Derg 2003; 11 (4): 235-41.

11 Kurosawa S, Kato M. Anesthetics, immune cells, and immune response. J Anesth 2008; 22 (3): 263 77.
12 Kelbel I, Weiss M. Anaesthetics and immune function. Curr Opin Anaesthesiol 2001; 14 (6): 685-91.

13 Gitzelman CA, Mendoza-Sagaon M, Talamini MA, Ahmad SA, Pegoli W, Paidas CN. Cell-mediated immune response is better preserved by laparoscopy than laparotomy. Surgery 2000; 27 (1): 65-71

14 Gupta A, Watson I. Effects of laparoscopy on immune function. Brit J Surg 2001; 88 (10): 12961306.

15 Fuchs D, Weiss G, Wachter H. Neopterin, biochemistry and clinical use as a marker for cellular immune reactions. Int Arch Allergy Imm 1993; 101: 1-6.

16 Wachter H, Fuchs D, Hausen A, Reibnegger G, Weiss G, Werner ER, Werner-Felmayer G. Neopterin, Biochemistry, Methods, Clinical Applications. Walter de Gruyter, Berlin, 1992.

17 Müller TF, Vogl M, Neumann MC, Lange H, Grimm M, Müller MM. Noninvasive monitoring using serum amyloid A and serum neopterin in cardiac transplantation. Clin Chim Acta 1998; 276: 63-74.

18 Hamerlinck FF, van Gool T, Faber WR, Kager PA. Serum neopterin concentrations during treatment of leishmaniasis: useful as test of cure? FEMS Immunol Med Mic 2000; 31-4.

19 Fuith LC, Czarnecki M, Mitterschiffthaler G, Wachter H, Fuchs D. Abdominal hysterectomy induces immune activation. Pteridnes 1997; 8: 1720.

20 Marfella A, Bilancio A, Polese C, Iodice F, Edmondo C, Cerasuolo D, Esposito G, Zannoni V, Beneduce G. Urinary neopterin and kynurenine in patients submitted to surgical stress with different inhalational anesthetics, halothane or isoflurane. Int J Immunopharmacol 1999; 21 (7): 423-33.

21 Yuksel O, Sahin TT, Girgin G, Sipahi H, Dikmen K, Samur O, Barak A, Tekin E, Baydar T. Neopterin, catalase and superoxide dismutase in females with benign and malignant breast tumors. Pteridines 2007; 18: 132-8.

22 Baydar T, Yuksel O, Sahin TT, Dikmen K, Girgin G, Sipahi H, Kurukahvecioglu O, Bostanci H, Sare M. Evaluation of neopterin excretion in intensive care unit patients. J Crit Care 2009; 24 (3): 318-21.

23 Sahin TT, Yuksel O, Girgin G, Sipahi H, Dikmen K, Azili C, Taneri F, Baydar T. Urinary neopterin in patients with thyroid disorders. J Endocrinol Inves 2009; 32 (2): 147-9.

24 Asci A, Baydar T, Cetinkaya R, Dolgun A, Sahin G. Evaluation of neopterin levels in patients undergoing hemodialysis. Hemodial Int 2010; 14: 240-6.

25 Gruber G, Lirk P, Amann A, Keller C, 
Schobersberger W, Hofman G. Neopterin as a marker of immunostimulation: an investigation in anaesthetic workplaces. Anaesthesia 2002; 57 (8): 747-50.

26 Homburger JA, Meiler SE. Anesthesia drugs, immunity, and long-term outcome. Curr Opin Anaesthesiol 2006; 19 (4): 423-8

27 Koksal M, Sayilgan C, Gungor G. Effects of sevoflurane and desflurane on cytokine response during tympanoplasty surgery. Acta Anaesth Scand 2005; 49 (6): 835-9.
28 Hönlinger M, Szava N, Fuchs D, Bernhart T, Mailath G, Wachter $\mathrm{H}$ : Hinweis für eine aktivierung des zellulären immunsystems bei operativer zahnentfernung. Z Stomatol 1992; 89: 487492.

29 Andersson C, Bernhart T, Hönlinger M, Mailath G, Wachter H, Fuchs D: Immune activation after apicoectomy: Comparison between patients with and without prophylactic antibiotic therapy. Pteridines 1993; 4: 192-194. 